\title{
La catálisis en México
}

\section{Jorge Noé Díaz de León,* Julia Aguilar Pliego**}

Debido al gran abanico de instituciones, líneas de investigación, investigadores y posgrados ofrecidos a lo largo del territorio nacional en catálisis y temas afines, se invitó a la comunidad catalítica en forma institucional y por medio de la página de la ACAT (http://www.acat.org.mx/) a contribuir en la realización de esta recopilación nacional de instituciones con actividades de investigación y desarrollo en catálisis. La respuesta a esta convocatoria ha sido exitosa pues se recibieron artículos de 16 instituciones, con lo que esperamos ofrecer una panorámica robusta de las diferentes actividades en catálisis que se realizan en cada entidad. Entre los aspectos destacados en este catálogo están: la presentación de cada entidad; líneas de investigación y desarrollo, tanto las desarrolladas como las que están en curso; infraestructura disponible; docencia y formación de recursos humanos; principales logros de sus miembros; perspectivas en el campo de investigación, además de los espacios digitales correspondientes a cada institución. En nuestro país existen numerosos centros de investigación, así como universidades en donde se realiza investigación relacionada con catálisis. Cabe mencionar, que, a nivel nacional, existe una gran variedad en temas de investigación.

A continuación, se presenta un análisis comparativo de las publicaciones a nivel mundial y las publicaciones de México. En la figura 1 se muestra una gráfica con la cantidad de publicaciones realizadas en el mundo relacionadas con el tema de catálisis. Mientras que en la figura 2 se pueden observar los valores de la búsqueda por país, seguimiento realizado en la plataforma Web of Science ${ }^{1}$ para 2016 y lo que va de 2017.

Los tópicos mostrados están relacionados con la actividad de los miembros de la ACAT, sin embargo, no es posible agrupar en una sola palabra toda la investigación que se realiza en México y la diversidad existente puede ir desde el estudio de la síntesis, la caracterización de catalizadores (metales, metales-soportados, óxidos, sulfuros, materiales mesoporosos nanoestructurados, zeolitas, materiales organometálicos, entre otros muchos) y su aplicación en diferentes tipos de reacción y de procesos, como: fotocatálisis, hidrodesulfuración (HDS), hidrodesoxigenación (HDO), hidrodenitrogenación (HDN), tecnología del hidrógeno, transformación de biomasa para obtención

* Universidad Nacional Autónoma de México-Centro de Nanociencias y Nanotecnología, Ensenada, Baja California.

** Área de Química Aplicada, Depto. C. B. UAM-A, Ciudad de México.

${ }^{1}<$ https://apps.webofknowledge.com/WOS_GeneralSearch_input.o?product=WOS\&search_

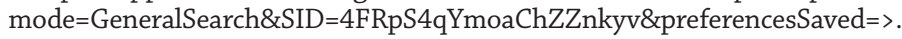




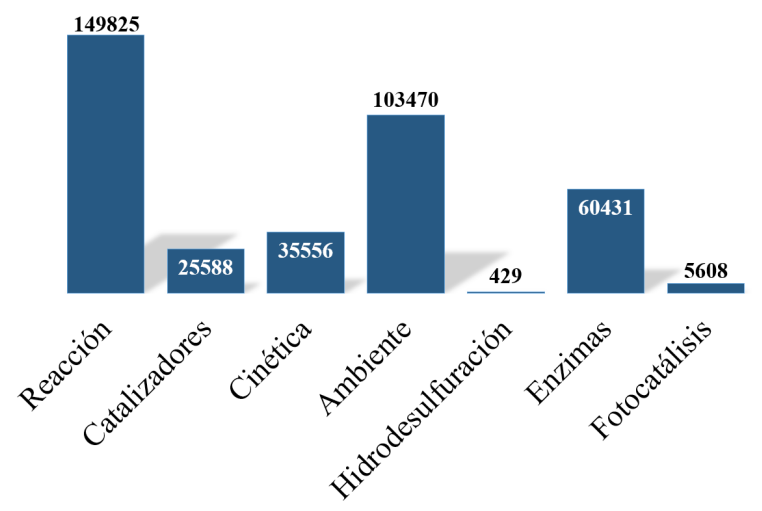

Figura 1. Número de publicaciones relacionadas con tópicos sobre catálisis en el mundo.

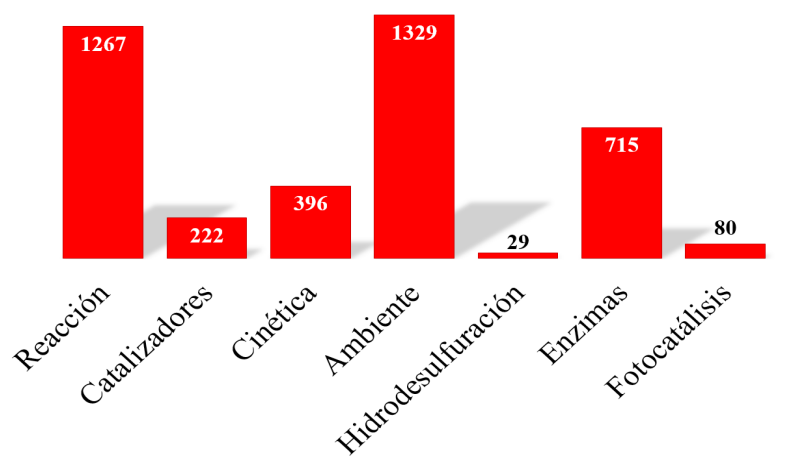

Figura 2. Número de publicaciones relacionadas con tópicos sobre catálisis en México.

de productos de alto valor agregado como biodiesel, bioturbosina, biogasolina, desulfuración oxidativa, desarrollo de procesos catalíticos, materiales nanoestructurados, catalizadores para celdas de combustible, catálisis enzimática, catálisis biomimética, entre otros. Lo que proporciona un gran abanico de líneas de investigación desarrolladas por los socios de la Academia. A pesar del gran dinamismo que se presenta en torno a la investigación, la docencia y la formación de recursos humanos en catálisis en México, no existe un posgrado en catálisis o una institución que se dedique exclusivamente al desarrollo de proyectos relacionados con esta disciplina, a diferencia de lo que ocurre en otros países, en los que sí hay institutos o centros dedicados exclusivamente al estudio de la misma. Es por esta razón que es importante mostrar la oferta nacional en investigación y desarrollo que tenemos en México en esta disciplina, tanto para la realización de estudios universitarios y 
de posgrado como para la colaboración interinstitucional. De la misma forma, se pretende que este catálogo permita al sector industrial tener una idea de a qué instituciones (investigadores) se puede acudir en busca de posibles soluciones para resolver sus problemas, sobre todo porque la catálisis trasciende a todos los aspectos de la cadena de producción industrial y tecnológica. El catálogo se presentará de acuerdo con las representaciones regionales en las que está organizada la ACAT, a saber, Baja California, Veracruz, Bajío, Norte, Occidente y Valle de México.

\section{Región Baja California}

Las instituciones en las que se realiza investigación y desarrollo en catálisis en esta demarcación son el Centro de Graduados e Investigación en Química del Instituto Tecnológico de Tijuana (CGIQ-ITT), el Centro de Nanociencias y Nanotecnología de la Universidad Nacional Autónoma de México (CNyNUNAM), el Centro de Investigación Científica y de Educación Superior de Ensenada (CICESE) y la Universidad Autónoma de Baja California (UABC) en sus campus Tijuana y Ensenada (véase la figura3).

En estas instituciones se están desarrollando diversas temáticas que van desde la catálisis homogénea hasta la producción industrial de catalizadores. Estas temáticas de investigación, así como los académicos asociados a éstas están descritas en una de las contribuciones que aparecen en este número especial. En esta región se cuenta con varios reactores de laboratorio por lotes y de flujo continuo para reacciones diversas como deshidratación de alcoholes, fotocatálisis o reacciones de desplazamiento de vapor de agua, destaca una planta para la evaluación de catalizadores de hidropurificación a escala de banco. Asimismo, se cuenta con un microscopio JEOL JEM-2100F (STEM) con cañón de electrones tipo emisión de campo-Schottky, con modo barrido, adquisición digital de imágenes, análisis químico por medio de espectroscopía de dispersión de energía (EDS), se pueden realizar mapas y espectroscopía de pérdida de energía de electrones (EELS). Así como un equipo

Figura 3. Entidades en las que se realiza catálisis de la Región Baja California.

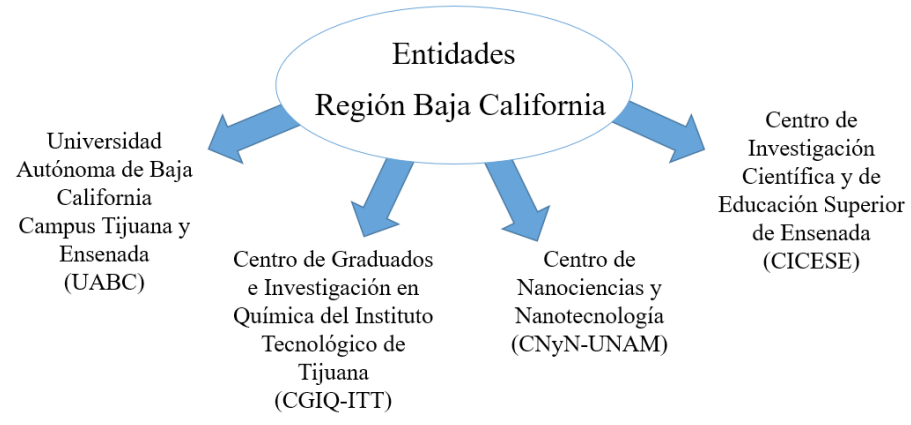


SPECS para análisis de espectroscopía fotoelectrónica de rayos X (XPS) que cuenta con una cámara de tratamientos a alta temperatura y presión, así como un analizador hemisférico con resolución angular. Además, en estas instituciones se ofrecen alrededor de 7 programas para estudios de licenciatura, maestría y doctorado que involucran temas de catálisis. Esta región cuenta con más de 20 miembros activos afiliados a la ACAT y su representante es el Dr. Jorge Noé Díaz de León.

\section{Región Veracruz}

Comprende las actividades de investigación y docencia realizadas en los laboratorio multidisciplinarios de la Universidad Veracruzana (UV) ubicados en sus cinco campus Xalapa, Coatzacoalcos-Minatitlán, Orizaba-Córdoba, Poza Rica-Tuxpan, y Veracruz-Boca del Río relacionadas con catálisis y nanomateriales diseñados para este propósito. Como es sabido, esta región del país es importante por su riqueza petrolera, donde, en general, se realiza investigación en diseño, síntesis y caracterización de catalizadores ácidos para la obtención de biocombustible a partir de biomasa, caracterización de residuos agrícolas para su uso como material catalítico, eliminación de microcontaminantes en aguas mediante procesos avanzados de oxidación, degradación de azocompuestos mediante procesos catalíticos, simulación de procesos, procesos y sustantibilidad y procesos de superficie, entre otros. Aquí se tienen técnicas de caracterización como espectroscopía de UV-vis, calorimetría diferencial de barrido, espectroscopía de infrarrojo, por mencionar algunos.

Además, en esta región se encuentra la unidad de Servicios de Apoyo en Resolución Analítica (SARA), Dependencia de la Dirección General de Investigaciones, creada mediante la participación activa de un grupo de investigadores de la Universidad Veracruzana, con el fin de ofrecer servicios de alta tecnología a profesionales de la investigación.

\section{Región Bajío}

Aquí se encuentran los estados de Querétaro, San Luis Potosí y Guanajuato. Las entidades que tienen miembros de la ACAT en estos estados son la Universidad Autónoma de Querétaro, la Universidad Autónoma de San Luis Potosí, el Instituto Potosino de Investigación Científica y Tecnológica, la Universidad de Guanajuato (UGTO) y el Instituto Tecnológico de Celaya. Sus miembros están enfocados en el análisis y preparación de catalizadores creados a partir de nuevos materiales como el grafeno, nanotubos de carbono y/o compuestos organometálicos. Estos materiales se analizan en reacciones de fotocatálisis, sensores de gases, deshidratación de alcoholes, oxidación de $\mathrm{CO}$, carbonilaciones de metanol, hidrogenación de $\mathrm{CO}_{2}$, deshidrogenación selectiva de aminas, entre otras. En esta región se cuenta con reactores hidrotérmicos asisti- 


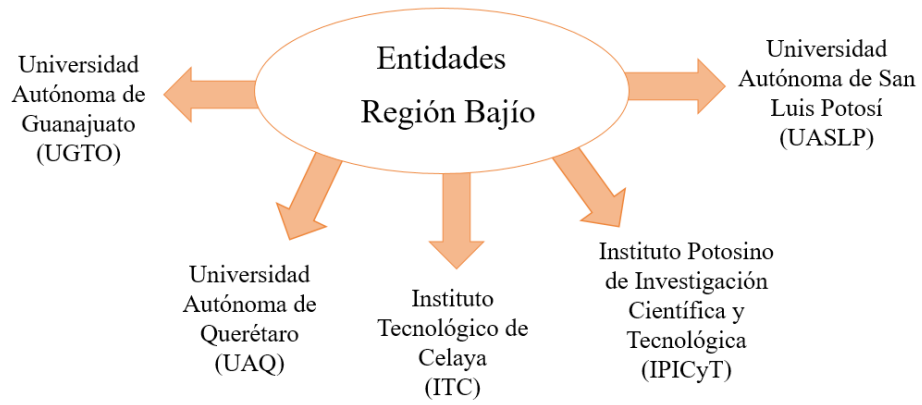

Figura 4. Entidades en las que se realiza catálisis de la Región Bajío.

dos por microondas, sistemas para síntesis fotoasitida de nanopartículas metálicas con lámparas LEDs a diferentes longitudes de onda y ultravioletavisible (UV-vis). Destacan los estudios de medición de carbono orgánico total, espectroscopías infrarrojo (IR) y UV-vis in situ que son ofrecidos a diversas instituciones. Así como el acceso al Laboratorio Nacional de Luz Sincrotron de Brasil en el que se realizan experimentos de espectroscopía de absorción de rayos $\mathrm{X}$ (EXAFS, por sus siglas en inglés) y absorción de rayos $\mathrm{X}$ cercana al borde de absorción (XANES, por sus siglas en inglés). En esta zona hay 31 miembros afiliados y su representante es la Dra. Esthela Ramos Ramírez.

\section{Región Norte}

Esta área engloba los estados de Nuevo León, Coahuila y Tamaulipas. Las entidades en las que se realiza catálisis o actividades relacionadas en estos estados son la Universidad Autónoma de Nuevo León (UANL), el Centro de Investigación en Química Aplicada (CIQA) y el Instituto Tecnológico de Ciudad Madero. Las investigaciones de esta región se enfocan en la síntesis de óxidos metálicos, materiales mesoporosos tipos SBA- 15 y zeolitas, todos ellos dopados con metales de transición como el Fe, Zn, Mn entre otros. Estos materiales se utilizan para oxidación catalítica de fenol y degradaciones fotocatalíticas de tricloroetileno o ácido salicílico, entre otros. Asimismo, se realiza catálisis homogénea aplicada a la síntesis de monómeros acrílicos y estirénicos, con y sin grupos polares, para la obtención de homopolímeros y copolímeros con estructuras bien definidas. Asimismo, se tiene amplia experiencia en la polimerización de olefinas con sistemas basados en zirconocenos (soportados, para trabajos en fase gaseosa, y no soportados) y polimerización de éteres vinílicos. ${ }^{2}$ En esta región se cuenta con equipos de caracterización

\footnotetext{
${ }^{2}<$ http://www.ciqa.mx/index.php/investigacion/80-sintesis-de-polimeros/42-proyectos-endesarrollo-sp>.
} 


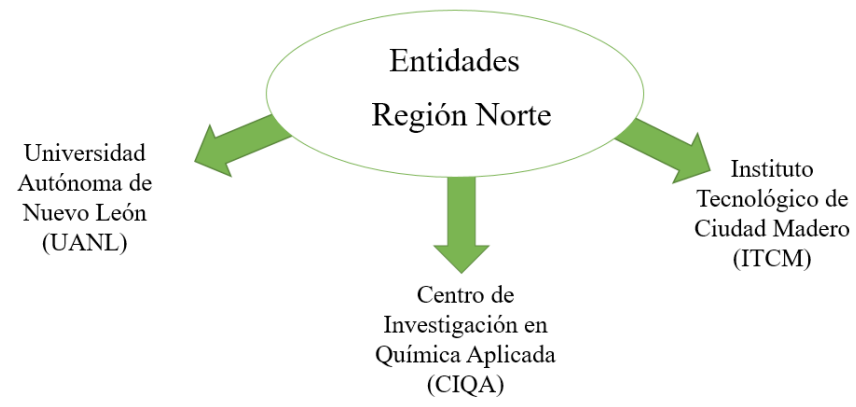

Figura 5. Entidades en las que se realiza catálisis de la Región Norte.

tales como XPS, HRTEM, microscopía electrónica de barrido (SEM, por sus siglas en inglés) modelo Nescope 6000 (JEOL), espectroscopía FTIR y en especial acceso a resonancia magnética nuclear (NMR, por sus siglas en inglés) para sólidos en colaboración con las Universidades de The City University of New York y Clemson University, en Estados Unidos de América.

Se cuenta con diversos reactores por lotes y empacados, pero destaca un reactor solar de tipo captador parabólico compuesto $(\mathrm{CPC})$. En esta región hay 22 miembros afiliados y su representante es el Dr. Javier Rivera de la Rosa.

\section{Región Occidente}

Incluye los estados de Michoacán, Jalisco y Colima. Sin embargo, las actividades de investigación en el área de catálisis en esta área son realizadas principalmente en la Universidad Michoacana de San Nicolás de Hidalgo (UMSNH) en la Facultad de Ingeniería Química (FIQ). Gracias a los apoyos recibidos del Instituto de Investigaciones Metalúrgicas de la UMSNH, las actividades de in-

Figura 6. Entidades en las que se realiza catálisis de la Región Bajío.

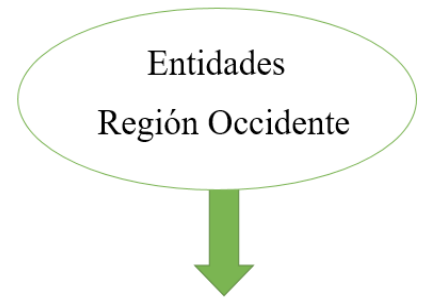

Universidad

Michoacana de San

Nicolás de Hidalgo

(UMSNH) 
vestigación fueron posibles y continúan siendo apoyadas en la actualidad. Esto se debe a que dicho instituto cuenta con infraestructura como los equipos de microscopía electrónica, difracción de rayos X, espectroscopía de infrarrojo. Asimismo, las líneas de investigación relacionadas con la catálisis en esta institución son fuentes alternas de energía, hidrotratamiento, degradación de contaminantes por medio de la fotocatálisis, reformación del metano tradicional y en seco, síntesis del amoniaco, uso de materiales naturales y de desecho como catalizadores, hidrogenaciones e isomerizaciones. Los tipos de materiales catalíticos usados son: metales, óxidos, sulfuros, fosfuros, nitruros y carburos metálicos, y de éstos se realiza la síntesis, caracterización y prueba de catalizadores en reacciones químicas, las cuales se llevan a cabo en diferentes tipos de reactores. Asimismo, se planea a futuro estudiar reacciones con biomasa para producción de biodiesel entre otras más, que pueden leerse en el capítulo concerniente a esta región. El representante en esta demarcación ante la ACAT es el Dr. José Luis Rico Cerda, y cuenta con 12 miembros afiliados.

\section{Región Valle de México}

Es donde se encuentra la mayor cantidad de instituciones con miembros de la ACAT, siendo alrededor de 50. Esta región comprende el estado de Hidalgo, el Estado de México y a la Ciudad de México. Las instituciones en las que se realiza catálisis son la Universidad Autónoma del Estado de Hidalgo (UAEH), la Universidad Autónoma del Estado de México (UAEM), el Instituto Nacional de Investigaciones Nucleares (ININ), Universidad Nacional Autónoma de México (UNAM), Instituto Mexicano del Petróleo (IMP), Instituto Politécnico Nacional (IPN) y la Universidad Autónoma Metropolitana (UAM) (véase la figura 7). Algunas de las instituciones mencionadas cuentan con diversas unidades, centros e institutos en los que se realiza investigación relacionada con

Figura 7. Entidades en las que se realiza catálisis de la Región Valle de México.

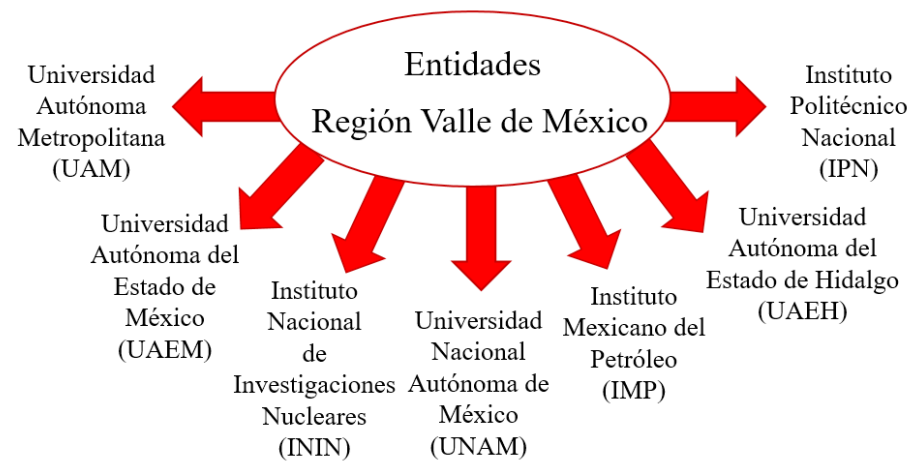




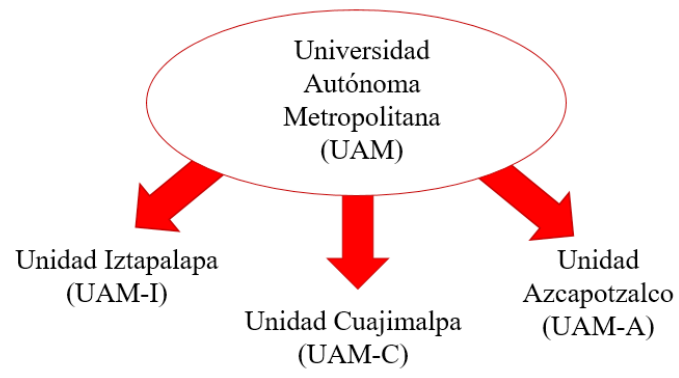

Figura 8. Unidades de la UAM en las que se realiza investigación en catálisis.

catálisis, por ejemplo, la UAM que tiene 3 unidades: la Unidad Azcapotzalco, la Unidad Cuajimalpa y la Unidad Iztapalapa (figura 8). A su vez, en la UNAM se realiza investigación y desarrollo en catálisis en el Instituto de Física (IFUNAM), el Instituto de Química (IQ-UNAM), el Instituto de Ingeniería (IIUNAM), el Instituto de Investigaciones en Materiales (IIM-UNAM), la Facultad de Química (FQ-UNAM) y el Centro de Ciencias Aplicadas y Desarrollo Tecnológico (CCADET-UNAM) (figura 9). Por su parte, el IPN cuenta con la Escuela Superior de Ingeniería Química e Industrias Extractivas (ESIQIE).

Esta región es muy compleja de describir pues es la que más instituciones agrupa. En general, hay proyectos relacionados con cada ámbito de la catálisis como la producción de nanocatalizadores para el control de la contaminación, síntesis y caracterización de materiales a base de nanopartículas para la producción fotocatalítica de $\mathrm{H}_{2}$, degradación de compuestos orgánicos volátiles, síntesis, formulación, caracterización y evaluación de catalizadores para hidrodesulfuración profunda de diésel y gasolina, síntesis y formulación de catalizadores para la desintegración catalítica fluida (FCC, por sus siglas en inglés), reformación e isomerización de gasolinas, síntesis y formulación de catalizadores para la producción de etileno, estudio de la estructura y propiedades catalíticas de nanopartículas con aplicación en medio am-

Figura 9. Instituciones de la UNAM en las que se realiza investigación en catálisis.

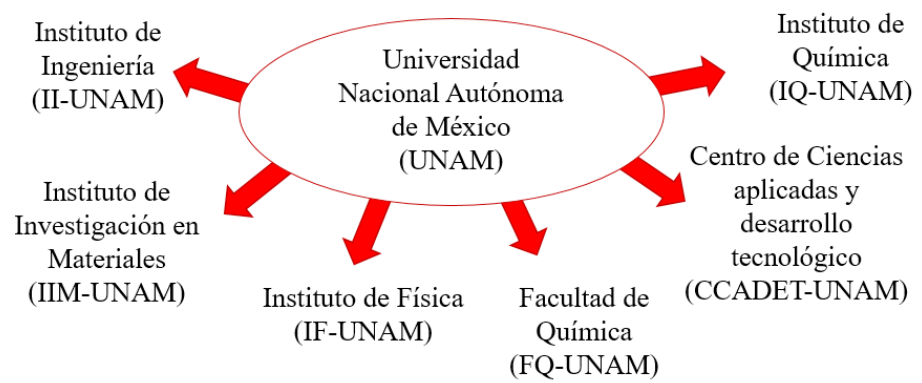


biente, energía y química fina, transformación y/o captura de $\mathrm{CO}_{2}$, aminación reductiva asimétrica de cetonas, nanotubos de carbono y grafeno para la generación de $\mathrm{H}_{2}$, aplicación de diferentes materiales catalíticos en reacciones con biomasa, entre muchos otros. Como es de esperarse en esta área, se cuenta con una gran variedad de equipos de caracterización y de evaluación catalítica tanto a nivel microrreacción como a nivel planta piloto, mismos que serán descritos a detalle en las contribuciones correspondientes.

La representante de esta región es la Dra. Martha Leticia Hernández Pichardo, y, además, la mayoría de los miembros del consejo directivo de la ACAT pertenecen también a esta región. La Dra. Julia Aguilar Priego de la UAM-A es su actual presidenta, el Dr. Rodolfo Zanella Specia del CCADET es el vicepresidente, las doctoras Dora Alicia Solís Casados y Reyna Natividad de la UAEM secretaria y vocal, respectivamente, y la Dra. Aída Gutiérrez Alejandre de FQ-UNAM es la tesorera. Asimismo, el Dr. José Antonio de los Reyes Heredia de la UAM-I es el actual presidente de la FISOCAT (Federación Iberoamericana de Sociedades de Catálisis).

Por último, cabe mencionar la existencia de algunas instituciones en las que se llevan a cabo actividades sobre catálisis como la Universidad Juárez Autónoma de Tabasco (UJAT) o la Unidad de Energía Renovable del Centro de Investigación Científica de Yucatán (UER-CICY) mismas que están en proceso de formar parte de la ACAT como una nueva región. 
